\title{
Delayed action does not always require the ventral stream: A study on a patient with visual form agnosia
}

\author{
Constanze Hesse $e^{1} \&$ Thomas Schenk ${ }^{2}$
}

$16 / 01 / 2014$

keywords: perception-action model, visual form agnosia, delay, posting, DF

RUNNING TITLE: "Patient DF posting after delay"

Correspondence should be addressed to:

Dr. Constanze Hesse

School of Psychology

University of Aberdeen

King's Campus

AB24 3FX

United Kingdom

e-mail: c.hesse@abdn.ac.uk

phone: ++44(0)19133404 47

\footnotetext{
${ }^{1}$ School of Psychology, University of Aberdeen, UK

${ }^{2}$ Neurology, University of Erlangen-Nuremberg, Germany
} 


\section{Abstract}

It has been suggested that while movements directed at visible targets are processed within the dorsal stream, movements executed after delay rely on the visual representations of the ventral stream (Milner and Goodale, 2006). This interpretation is supported by the observation that a patient with ventral stream damage (DF) has trouble performing accurate movements after a delay, but performs normally when the target is visible during movement programming. We tested DF's visuomotor performance in a letter-posting task whilst varying the amount of visual feedback available. Additionally, we also varied whether DF received tactile feedback at the end of each trial (posting through a letter box vs. posting on a screen) and whether environmental cues were available during the delay period (removing the target only vs. suppressing vision completely with shutter glasses). We found that in the absence of environmental cues patient DF was unaffected by the introduction of delay and performed as accurately as healthy controls. However, when environmental cues and vision of the moving hand were available during and after the delay period, DF's visuomotor performance was impaired. Thus, while healthy controls benefit from the availability of environmental landmarks and/or visual feedback of the moving hand, such cues seem less beneficial to DF. Taken together our findings suggest that ventral stream damage does not always impact the ability to make delayed movements but compromises the ability to use environmental landmarks and visual feedback efficiently.

keywords: perception-action model, visual form agnosia, delay, posting, patient DF 


\section{Introduction}

The distinction between immediate and memory-guided actions plays an important role in Goodale and Milner's influential perception-action model (Goodale and Milner, 1992; Milner and Goodale, 1995, 2006). According to this model, visual information is processed differently depending on the purpose for which the information is acquired: In order to identify or recognise objects, information is processed within the ventral stream (vision for perception), whereas for the visual guidance of actions, information is processed within the dorsal stream (vision for action). In order to suit the different purposes of action and perception, it was suggested that both visual streams operate on different time scales (Goodale et al., 2005; Goodale et al., 2004; Milner et al., 2001; Rossetti, 1998). For action planning, the relative position between target and observer has to be taken into account. Since in natural conditions observers are moving, these relative positions change constantly. Accordingly, it was proposed that information in the dorsal stream is not stored over longer periods of time. In contrast, for object identification and recognition, information in the ventral stream has to be stored independently of view-point over longer time spans. Following this argument, it was suggested that actions directed to visible targets are guided by the dorsal stream whereas actions executed after a delay (delayed actions or memoryguided actions) have to rely on ventral stream information, thus causing alterations in movement kinematics (Goodale et al., 2005; Goodale et al., 2004; Hu et al., 1999; Westwood and Goodale, 2003; Westwood et al., 2003).

Originally, it was assumed that visual information in the dorsal stream remains available for a maximum time of $2 \mathrm{sec}$ (Goodale et al., 1994). However, more recently it has been suggested that the dorsal stream is in fact only engaged in movement programming if vision is available at the moment when the movement is initiated (Goodale et al., 2005; Goodale and Westwood, 2004; Goodale et al., 2004; Westwood and Goodale, 2003). According to this "real-time view 
of motor programming", action programming does not occur before the decision to move has been made. Specifically, it has been hypothesised that the dorsal stream can only supply the relevant visual information if this information is present on the retina during action programming. In other words, if visual information is unavailable during the programming phase of the movement, the action system has no access to dorsal input and instead has to rely on visual information from the ventral stream resulting in qualitatively different movements.

Recent research on the topic, primarily investigating grasping movements, has called this real-time view (i.e. the suggestion that the dorsal stream is amnestic) into question. The real-time view of action makes three clear predictions: (1) the kinematics of movements performed after delay will be considerably different from the kinematics of movements that are programmed whilst the target object is visible; (2) the introduction of a delay period will shift the neuronal activity from the dorsal to the ventral system; (3) damage to the ventral stream will selectively impair the patient's ability to carry out accurate movements after delay. The first prediction was examined by Hesse and Franz $(2009,2010)$ and they found that in healthy participants grasping kinematics did not change abruptly depending on whether visual information is present during the movement programming phase or not (Hesse and Franz, 2009, 2010). Instead, the authors suggested that there is a gradual (exponential) decay of visuomotor information over time (Hesse and Franz, 2010; for a different view see, Hu et al., 1999; Westwood et al., 2001). The second prediction was examined in a number of functional imaging studies. These studies revealed that even after a delay of up to $12 \mathrm{~s}$, activity can be found in the dorsal stream area when simple reaching and grasping movements have to be performed (Connolly et al., 2003; Fiehler et al., 2011; Himmelbach et al., 2009; Lacquaniti et al., 1997). Based on these findings, it has been suggested that the changes in movement kinematics after delay might be attributed to an information decay occurring in both visual streams (Hesse and Franz, 2009), or alternatively that there might be 
a much more gradual shift from dorsal to ventral processing over time (Himmelbach and Karnath, 2005).

What about the third prediction? The prediction that selective ventral stream damage will lead to a selective deficit of delayed actions was examined in patient DF, who has sustained bilateral damage to her ventral stream (Milner et al., 1991). Until now, this prediction has received support from all relevant studies (Goodale et al., 1994; Milner et al., 1999; Milner and Goodale, 2006; Rossit et al., 2010). The observed dissociation between DF's intact ability to perform immediate actions and her impaired ability to perform accurate delayed actions provides therefore the most compelling evidence for the assumption that the dorsal and the ventral stream work on different time scales. Hence, if we want to challenge the hypothesis that delayed actions critically depend on ventral stream processing, we need to provide an alternative explanation for the finding that DF's visuomotor performance is impaired after delay.

In our alternative account we assume that visual information decays generally over time (no matter where it is represented) resulting in less accurate movements with longer delays (for similar view see also, Elliott et al., 1990). In particular, the memory decay will be associated with an increased uncertainty about the properties of the target object such as its position, size or orientation. Healthy participants are able to partly compensate for this information decay by using other available (sensory) information such as visual landmarks. Previous research suggests that the availability of visual landmarks facilitates not only visually guidedmovements but also movements performed after delay (Conti and Beaubaton, 1980; Hay and Redon, 2006; Krigolson and Heath, 2004; Lemay et al., 2004). That is, if visual background information (for example, four illuminated LEDs positioned in a square surrounding the target locations) is presented, reaches are more accurate and less variable than in conditions in which the same targets are presented on an empty (dark) visual background (Krigolson and 
Heath, 2004). Based on these findings it was argued that the availability of contextual information surrounding a target (allocentric cues) in combination with vision of the moving limb (egocentric cues) considerably enhances the accuracy of (memory-guided) movements. In situations in which no environmental cues are provided, the only information available in order to point accurately is the remembered position of the target in relation to your own body (egocentric information). However, by providing visual landmarks during the delay period the target can be coded relative to these landmarks (e.g. about $10 \mathrm{~cm}$ to left of the LED). Coded this way, the target position can still be recalled accurately, even after a delay of a few seconds, provided the visual landmarks are still available during and after the delay period. Hence, environmental cues help to form a more accurate and stable memory representation of the object's properties. If we assume that this is how healthy participants handle the problem of delayed action, how can we explain why DF has difficulties in performing accurate movements after delay? The answer to this question is relatively straightforward. The effective use of landmarks from the visual environment requires that the observer can code the properties of the visual target relative to the certain landmarks. This is called scene-based or allocentric coding and it has been demonstrated in previous studies that this type of coding is specifically impaired after ventral stream damage (Dijkerman et al., 1998; Schenk, 2006). Consequently, we hypothesise that DF cannot use visual landmarks to reduce delay-induced uncertainty about the target position.

In our opinion, this alternative account could potentially explain the findings reported in two out of three studies which tested DF's performance after delay. In those two studies environmental information remained available during the delay period (Milner et al., 1999; Rossit et al., 2010). In both studies, testing DF's eye and hand movements respectively, only the target was removed from view during the delay period while the experimental setup (i.e. the monitor or target panel on which the stimuli were presented) remained visible to all 
subjects. In contrast, in the third study in which Goodale and colleagues (1994) showed that DF has problems performing grasping movements after a delay of $2 \mathrm{sec}$, environmental information was not available (i.e. participants and patient DF kept their eyes closed during the delay period). However, it is important to note that in this experiment, all delayed movements were performed pantomimed, meaning that no real object was presented and grasped at the end of the trial. Therefore, the study also does not provide conclusive evidence regarding the question of whether the temporal delay, or the fact that no object was presented caused the observed alterations in movement kinematics. This is critical for two reasons: Firstly, it has repeatedly been shown that DF has problems with pantomiming actions, such as grasping or posting, even if the target object remains visually present (Goodale et al., 1991; Milner and Goodale, 2006; Milner et al., 1991). Secondly, recent research seems to suggest that the presence of tactile feedback is a crucial factor in DF's ability to generate normal grasping movements (Schenk, 2012a, b; for different interpretation see, Milner et al., 2012; Whitwell and Buckingham, 2013) .

Thus, at a first glance it appears that our account could in fact provide a valid alternative explanation for the finding that patient DF's visuomotor performance is impaired after delay as reported in previous studies (Goodale et al., 1994; Milner et al., 1999; Rossit et al., 2010). However, this gives rise to the following questions: Firstly, how exactly does our account differ from the predictions made by the perception-action model? And secondly, how can we test which of the two accounts is correct? The first question is important as there are some similarities between the assumptions of the perception-action model and our view. Both accounts assume that observer-relative or egocentric coding becomes increasingly unreliable when a delay is introduced, simply because movements made by the observer may render such egocentric relationships unstable. Furthermore, both accounts also assume that allocentric coding based on visual landmarks provides a more stable reference frame for 
delayed actions. But this is where the similarities end. The perception-action model further assumes that the dorsal stream is incapable of maintaining visual information for more than a few milliseconds (real-time view) or at best 2 seconds. This means any delay in excess of a couple of seconds will force the visual system to make use of the perceptual (and presumably allocentric) information that is available in the ventral stream. Thus, according to the perception-action model any delay between the visual presentation of a target object and an action initiated toward that object will inevitably induce a shift from dorsal to ventral stream control of action and therefore will inevitably impair DF's visuomotor behaviour independent of the specific task conditions. In contrast to the perception-action model, we assume that both visual streams operate on similar time scales and that both streams can recall visual information if necessary after delays of more than a few seconds. Furthermore, we assume that the use of allocentric coding and thus ventral stream processing is not mandatory but strategic, i.e. participants will make increased use of allocentric coding when allocentric information is more reliable. This is particularly the case in situations in which the visual target is extinguished prior to motion onset but visual landmarks are still present. We therefore predict that DF's visuomotor behaviour after delay is only abnormal when visual landmarks are present during the visuomotor response.

In our study, we examined DF in a classical letter-posting paradigm. In Experiment 1, we examined the effect of various delays on her visuomotor performance and compared her performance under delay-conditions with her pantomime performance. In Experiment 2, we directly examined the role of visual landmarks on DF's behaviour in delayed and nondelayed condition and controlled for the potentially confounding effect of tactile feedback. We chose the letter-posting task as our experimental paradigm as this is a classical visuomotor task in which patient DF has been tested several times previously (Milner and Goodale, 2006; Milner et al., 1991). Therefore, we could be sure that the task is appropriate 
to reliably reveal perception-action dissociations, if present. As the task had never been tested in a delayed paradigm before, the experiment constitutes a logical addition to the existing literature and a suitable way to test the reliability of the claim that DF is impaired in all visuomotor tasks as soon as a delay is introduced. As pointed out above, this claim seems to be built upon relatively little experimental evidence and very few experimental paradigms (Goodale et al., 1994; Milner et al., 1999; Rossit et al., 2010) .

\section{Experiment 1: Letter-Posting (classical setup)}

\subsection{Methods}

\subsubsection{Participants}

Patient DF: A patient with visual form agnosia (DF) participated in the experiment. Patient DF suffered a carbon monoxide intoxication in 1988 that led to extensive bilateral brain damage (Milner et al., 1991). Magnetic resonance imaging revealed that her brain lesions are mostly confined to the ventral lateral occipital cortex (LOC), comprising in both hemispheres parts of Brodmann areas 18 and 19, but largely excluding V1 and the fusiform gyrus (James et al., 2003). Patient DF has trouble discriminating between different visual shapes, orientations and distances causing poor object recognition. Her luminance, colour, and texture perception are normal (Milner et al., 1991). DF wore glasses correcting for a slight presbyopia. At the time of testing she was 57 years old.

Control group: Ten female, right-handed and age-matched (mean age: 54 years, age range: 48-61 years) control participants were tested. All participants had normal or corrected to normal visual acuity and no history of neurological problems. All experiments were undertaken with the understanding and written consent of each participant and approved by the local ethics committee. Control participants were paid $£ 8$ per hour. 


\subsubsection{Apparatus and Stimuli}

Figure 2 shows the experimental setup for the letter-posting task. The posting device, which was placed on the table top, consisted of a vertical panel $(60 \mathrm{~cm} \times 60 \mathrm{~cm})$ on which a wooden disk (diameter: $40 \mathrm{~cm}$ ) with a centered slot $(12 \mathrm{~cm} \mathrm{x} 3 \mathrm{~cm})$ could be mounted. The disk was plugged on the vertical panel using pins such that the orientation of the slot could be changed easily. The slot was presented in 4 different orientations: $0^{\circ}$ (vertically), $22.5^{\circ}, 45^{\circ}$ and $67.5^{\circ}$ rotated in clockwise direction from the vertical orientation. A red plastic card with a size of 8 $\mathrm{cm} \times 8 \mathrm{~cm}$ (width $1 \mathrm{~cm}$, weight $72 \mathrm{~g}$ ) served as posting object. The resting position of the card was marked by a wooden cube $(4 \mathrm{~cm} \times 4 \mathrm{~cm} \mathrm{x} 4 \mathrm{~cm})$ on top of which the plastic card was placed before each trial at a $90^{\circ}$ orientation. As the target slot was never present at $90^{\circ}$ (horizontal orientation), each trial required that participants turned the card to match the orientation of the slot.

Two infrared light-emitting diodes (IREDs) were attached to the front edge of the card and their position was recorded by an Optotrak 3020 system (Northern Digital Incorporation, Waterloo, Ontario, Canada) at a sampling rate of $200 \mathrm{~Hz}$. Prior to the experiment, a calibration procedure was used to align the Cartesian coordinate system $(\mathrm{x}, \mathrm{y}, \mathrm{z})$ of the Optotrak system to the table top plane. During the experiment, participants wore liquidcrystal shutter goggles (PLATO Translucent Technologies, Toronto, Ontario; Milgram, 1987), which rapidly suppress vision by changing from a transparent to a translucent state (allowing light but no visual detail to pass through the lenses). Experiments were programmed in MATLAB using a custom-built Optotrak Toolbox (Franz, 2004).

\subsubsection{Procedure}


Participants sat on an adjustable chair within a lit room looking straight at the posting device and the target slot. Participants started each trial holding the card in their dominant right hand at the starting position. The straight-line distance between starting position and the centre of the slot was approximately $40 \mathrm{~cm}$, and the start position was aligned to the participant's body midline.

\section{Insert Figure 1 about here}

At the beginning of each trial, the shutter glasses turned translucent and the experimenter rotated the disk with the slot to a certain orientation. Note that the slot orientation was changed by removing the disk from the device, turning it (in the air) to the corresponding orientation and placing it back on the device. Therefore, there was no auditory information which would help the participants to guess how much the disk was rotated in-between trials. In trials, in which the orientation was the same as in the previous trial, the experimenter still removed the disk and placed it back in the same orientation. After the disk was re-attached with its slot oriented at the desired orientation, the experimenter initiated the trial manually by pressing a key. Subsequently, the shutter glasses changed to a transparent state for a preview period of $1.5 \mathrm{sec}$. Participants were instructed to view the slot during the preview period but to wait with their movement until an auditory go-signal (duration of $100 \mathrm{msec}$ ) had been presented.

All participants performed four different blocks of posting trials that differed in the amount of visual feedback available during movement execution (Figure 1). In the "closed-loop" (CL) condition, the auditory signal directly followed the preview period and the shutter glasses remained transparent for another $4 \mathrm{sec}$, such that participants could see both the slot and their hand during posting. In the "open-loop after movement initiation" (OLM) condition, the preview period was followed by the go-signal and the shutter glasses turned translucent as soon as the card was lifted from the starting position (i.e., after one of the markers had moved 
more than $10 \mathrm{~mm}$ away from the starting position in $\mathrm{z}$-direction). Therefore, the occlusion of vision was triggered by the movement of the card meaning that participants were still able to see the target slot during the movement programming phase (time between go-signal and movement initiation). In the "open-loop after start signal" (OLS) condition, the shutter glasses turned translucent at the same time as the go-signal was given. In both the OLM and the OLS conditions, participants could neither see the posting device nor their hand during posting. The only difference between the OLM and the OLS condition was therefore whether the target slot was visible during the movement programming phase or not. Finally, there was a "3s-delay condition" (3s-D) in which a $3 \mathrm{sec}$ delay was inserted between the preview period and the auditory go-signal. During the delay and the following posting movement the glasses remained translucent so that the participants had to remember the slot orientation for the duration of the delay and the following posting task. In all posting blocks, participants were instructed to put the card through the slot as accurately as possible.

Additionally, we introduced an orientation matching task (OM): For the orientation-matching task, participants were asked to move the card to the right side of the slot to a position marked on the table by a green paper cross (Figure 2B) and to match the orientation of the card to the orientation of the slot (similar to the pantomiming task conducted by Milner et al., 1991). Orientation matching was performed in a closed-loop vision condition meaning that the shutter glasses remained open during movement execution.

\section{Insert Figure 2 about here}

In all conditions, participants were allowed $4 \mathrm{sec}$ to execute the movement. After $4 \mathrm{sec}$ the Optotrak stopped measuring and in the CL-condition the shutter glasses closed. If the participant had not reached the target panel before this time limit was exceeded, the trial was classified as an error and was repeated later in the experiment at a random position within the 
trial-sequence (this happened only very occasionally when participants forgot to begin their movement). All conditions were performed in blocks of 32 trials ( 8 trials per slot orientation). The orientation of the slot varied randomly from trial to trial within each block. Each block was preceded by eight practice trials to familiarize the participant with the task. Thus, participants always knew in advance how much visual feedback was available during movement execution. This design was chosen for two reasons: Firstly, previous studies with DF also used a blocked design and we considered it important to replicate aspects of previous studies on this topic as faithfully as possible. Secondly, it has been shown that blocking vision conditions usually reveals larger differences between vision and no-vision trials than designs employing a random feedback schedule (randomized sequence of trials with and without visual feedback). It was found that with random feedback schedules performance in vision trials gets more similar to that in no-vision trials meaning that vision of the hand is no longer been used as efficiently (e.g., Whitwell and Goodale, 2009; Whitwell et al., 2008). Hence, using a blocked design provides a stronger test for the role of visual feedback and consequently failing to find a visual feedback effect in blocked conditions would provide stronger evidence to demonstrate that DF does not have to rely on online visual feedback to produce good posting performance.

Participants were instructed to post the card through the target slot as accurately as possible. No instructions were given as to the speed of initiation and the speed of the movement. The experimenter sitting next to the participant monitored the participants' movements to ensure that they followed instructions to put the card through the slot.

\subsubsection{Data analysis}

To calculate the card's velocity, we tracked the $3 \mathrm{D}$ position of the virtual mid-point between the two markers that were attached to the card and calculated the first time-dependent 
derivation of this position along the motion path. Movement onset was defined by a velocity criterion. The first frame in which the card velocity exceeded a velocity threshold of 0.025 $\mathrm{m} / \mathrm{sec}$ was taken as movement onset. In order to determine the adjustment of the card orientation to the slot orientation, we calculated the orientation of the card $2 \mathrm{~cm}$ before the target slot was reached (in z-direction, see Fig. 2A). We chose to compute the card orientation shortly before the target slot was reached to ensure that the measured orientation was not informed by tactile feedback that participants receive when they collide with the target panel. The orientation of the card as projected onto the plane of the slot-board facing the participant was calculated relative to the vertical (12 o'clock) position. The clockwise rotation was defined as positive. The orientation error was determined as the average absolute (unsigned) deviation of the card orientation from the actual slot orientation (absolute error) and as the averaged relative (signed) deviation of the card from the slot orientation (constant error). Furthermore, as it was shown that movements become more variable after delay, we also determined the variable error computed as the average standard deviation of the constant error. To calculate the orientation in the orientation-matching condition, we first determined the frame in which the card was moved furthest from the start position and then computed the card orientation at the sample with the lowest velocity occurring after the furthest distance from the start position was reached. We had to use this slightly more complicated set of criteria because the end position for the card was not as well-defined in the pantomime condition as in the posting conditions. For all statistical comparisons, we applied dissociation tests (Crawford and Garthwaite, 2005; Crawford et al., 2010) and modified t-tests specifically developed for single-case studies (Crawford and Howell, 1998).

\subsection{Results}

\subsubsection{Accuracy}


Our main dependent variable was the accuracy with which the card orientation was matched to the orientation of the target slot. First of all, it is important to note that in all four vision conditions neither patient DF nor the control participants failed to successfully post the card through the slot within the $4 \mathrm{sec}$ provided for movement execution. Figure 3 show the average orientation error measurements (A: constant error, B: absolute error and C: variable error) for each vision condition averaged over all slot orientations for DF and each control participant. The results suggest that DF had no trouble posting the card through the slot independent of the amount of visual feedback provided. However, if DF was asked to match the orientation of the slot without posting the card (pantomiming), her performance seemed to be impaired compared to control participants. To confirm this observation statistically, we conducted modified $t$-tests which were designed to compare an individual's test score against the test scores derived from small sample sizes (Crawford and Howell, 1998). These tests revealed that DF's posting performance did not differ significantly from the performance of the controls in all vision conditions (constant error: all $\mathrm{p}>.31$; absolute error: all $\mathrm{p}>.23$; variable error: all $\mathrm{p}>$.29). In fact, Figure 3 shows that DF's orientation errors tended to be smaller than the average errors made by the control participants in the reduced visual feedback conditions. However, in the pantomiming condition, DF's performance was significantly worse than that of the controls (constant error: $\mathrm{t}(9)=7.9, \mathrm{p}<.001$; absolute error: $\mathrm{t}(9)=13.7, \mathrm{p}<.001$; variable error: $\mathrm{t}(9)=8.3, \mathrm{p}<.001)$

To exclude the possibility that DF might have started to adjust the card to the slot orientation already during the preview period, we calculated the orientation of the card at movement onset. Please note that this possiblity is extremely unlikely as the card was placed (horizontally at $90^{\circ}$ ) at a wooden start cube between trials and the experimenter made sure that the card was not lifted before the presentation of the start signal. Results confirmed that across all slot orientations and vision conditions the card orientation at movement onset 
varied between $88.1^{\circ}$ and $89.5^{\circ}$. Thus, on average, there was less than $1.5^{\circ}$ of variation in the start orientations of the card between different slot orientations.

Finally, to examine the influence of the different visual conditions on the performance of the healthy control participants a repeated-measures ANOVA was performed. The analysis showed that the vision condition had a significant effect on movement accuracy (constant error: $F(3,27)=10.6, p=.006$, absolute error: $F(3,27)=17.3, p=.001)$, and movement variability (variable error: $\mathrm{F}(3,27)=28.7, \mathrm{p}<.001$ ). Post-hoc tests revealed that the absolute error and the variable error increased with decreased visual feedback. Apart from the difference between the OLS and 3s-D condition, all differences between vision conditions reached significance $(\mathrm{p}<.03)$. Regarding the constant error, post-hoc tests confirmed that participants showed a lower constant error in the CL-condition than in all other vision conditions (all $\mathrm{p}<.01$ ). However, there were no significant differences between the constant errors measured in the OLM, the OLS and the 3s-D condition (all $\mathrm{p}>.26$ ).

Insert Figure 3 about here

\subsubsection{Reaction time (RT) and Movement time (MT)}

In order to test whether DF's movement planning or movement execution times differed from those of the controls, we determined the RTs and MTs in all posting conditions averaged over the different slot orientations (c.f. Table 1). T-tests confirmed that neither DF's RTs nor her MTs were significantly prolonged compared to that of control participants (all $\mathrm{p}>.23$ ).

\section{Insert Table 1 about here}

\subsection{Discussion}

In this experiment we wanted to examine how well DF (a patient suffering from visual form 
agnosia caused by bilateral ventral stream damage) performs in a classical visuomotor task when visual feedback and environmental cues are unavailable during movement programming and when a delay is introduced between target presentation and movement initiation. Interestingly, we found that DF had no trouble performing the letter-posting task as accurately as the control participants after a delay. Her performance was not different from the performance of neurologically intact individuals when the amount of visual feedback was reduced in all four vision conditions tested. Most importantly, and in contrast with the predictions of the real-time view of motor programming, we did not find that DF's performance was dependent on the availability of visual information during the movement planning phase (Goodale et al., 2005; Goodale and Westwood, 2004; Goodale et al., 2004; Westwood and Goodale, 2003). To reiterate, according to the real-time view of motor programming, it would have been predicted that DF's performance would be similar to that of healthy controls in the CL and the OLM conditions (guided by dorsal stream), but be significantly impaired in the OLS and 3s-D conditions (guided by the ventral stream). In agreement with earlier studies, we observed that DF was unable to accurately pantomime the orientation of the target slot (Goodale et al., 1991; Milner and Goodale, 2006). Within the framework of the perception-action model, both types of movements (pantomimed and delayed) are assumed to depend on visual information from the perceptual ventral stream system and ventral-stream damage should thus affect both in a similar manner.

To summarise, Experiment 1 demonstrates clearly that delay per se does not impair DF's visuomotor performance. This confirms our prediction that DF performs normally after delay in situations in which no environmental (allocentric) visual information is available. This is certainly a surprising result that challenges a central assumption of the perception-action model, namely the assumption that success in visually-delayed action always requires an 
intact ventral stream. However, given the fact that previous studies found a detrimental effect of delays on DF's visuomotor behaviour (Goodale et al., 1994; Milner et al., 1999; Rossit et al., 2010), we aimed at further narrowing down the factors which determine whether or not DF's behaviour is impaired by introducing a delay between target presentation and action initiation. Specifically, we were interested in two possible factors: The role of tactile feedback and the role of environmental cues. In Experiment 1, participants were always able to use tactile feedback to learn whether their posting attempt had been successful or not: When they used the correct position and orientation for their card, it slipped unhindered through the slot, but when they misjudged the position or orientation of the slot they collided with the board. Therefore, Experiment 2 employed a "virtual slot", i.e. a white bar within a white circle presented on a computer monitor. Such a virtual slot offers no informative tactile feedback about posting accuracy. Instead, participants will always collide with the monitor and the tactile feedback will thus be the same regardless of how well participants match the slot orientation with their card. If DF relied on tactile feedback to compensate for the lack of visual guidance during the delay-period in Experiment 1, her performance should deteriorate significantly once the real slot is replaced by a virtual one. The second factor, which we will investigate in Experiment 2 is the role of environmental cues. Previous studies have repeatedly demonstrated that environmental (landmark) information (i.e. allocentric cues) in combination with vision of the moving limb (i.e. egocentric cues) increase the accuracy of memory guided movements (Conti and Beaubaton, 1980; Hay and Redon, 2006; Krigolson and Heath, 2004). In Experiment 1, no visual landmarks were available during the response period and thus healthy participants and DF performed alike. However, to examine whether the presence of visual landmarks during the response period can indeed explain why healthy participants sometimes perform superior to patient DF in delay conditions, we wanted to manipulate the presence of visual landmarks or environmental cues. Using a virtual-slot setup 
it is possible to remove just the target information by deleting the white bar from the screen while retaining all other visual information from the environment (e.g. the frame of the virtual disk as well as the frame of the monitor) and the moving hand. We will compare this condition directly to a condition in which both target and visual information from the environment are removed using LC shutter glasses. We predict that if DF cannot exploit such environmental cues, her performance might dissociate from that of healthy participants in conditions where environmental cues remain available during the movement and/or delay period.

\section{Experiment 2: Letter posting (virtual slot setup)}

\subsection{Methods}

\subsubsection{Participants}

Patient DF who suffers from visual form agnosia due to a bilateral damage in her ventral stream was tested in this experiment (see section 2.1.1 for details). At the time of testing she was 58 years old.

Control group: Ten female, right-handed and age-matched (mean age: 54 years, age range: 49-61 years) control participants were tested (4 of them had also participated in Experiment 1). All participants had normal or corrected to normal visual acuity and no history of neurological problems. All experiments were undertaken with the understanding and written consent of each participant and approved by the local ethics committee. Control participants were paid $£ 8$ per hour.

\subsubsection{Apparatus and Stimuli}

The setup was similar to the one used in Experiment 1. The wooden posting device was replaced with a 19" LCD monitor (HANNS.G HX191D, 1280 x 1024 pixel, 75 Hz), on which 
a virtual target slot was presented centrally (white bar on a black background) within a virtual white ring (representing the target disk of Experiment 1) with an inner diameter of $22 \mathrm{~cm}$ and an outer diameter of $23.5 \mathrm{~cm}$. The target bar had the same dimensions $(12 \mathrm{~cm} \times 3 \mathrm{~cm})$ as the slot used in Experiment 1 and could be presented in 5 possible orientations $\left(-22.5^{\circ}, 0^{\circ}\right.$ (vertically), $22.5^{\circ}, 45^{\circ}$ and $67.5^{\circ}$ ). The experiment was programmed in MATLAB using the Psychophysics Toolbox (Brainard, 1997; Kleiner, 2010).

\subsubsection{Procedure and Data analysis}

In most respects the procedures used in this experiment were similar to those in Experiment 1. Here we describe only those features that were different between experiments. To put it briefly, we manipulated two variables, each of those variables had two levels. Duration of vision was the first variable and the two options were open-loop viewing until movement initiation (OLM) or 3-second delay viewing (3s-D). The presence of environmental cues was the second variable: environmental cues could either be present $(\mathrm{EC}+)$ or absent (EC-). This yielded the following four task conditions:

OLM-EC-: Participants had a preview period of $1.5 \mathrm{sec}$ and were able to see the target and their visual environment until the time when they started their movement (after the start signal was presented). At movement onset the LC shutter glasses closed and occluded their vision of both the target and the surrounding environment.

3sD-EC-: Again, participants had a preview period of $1.5 \mathrm{sec}$, shutter glasses then closed and remained closed until the end of the trial. Three seconds after the closing of the shutterglasses a start-signal instructed the participants to begin their posting movement.

OLM-EC+: Participants had full vision of the target and their visual environment for the duration of the preview period and during the time they needed to initiate their movement after the presentation of the start-signal. At movement-onset the target slot disappeared from 
the screen but participants could still see the monitor and all the surrounding space (i.e. participants did not wear shutter glasses).

3sD-EC+: Participants had a preview period of 1.5 seconds. After this preview period the target slot disappeared, but participants could still see the monitor with its surrounding space. Three seconds later a tone instructed the participants to start with their posting movement.

The reader will notice that we only used a subset of the vision conditions that were tested in Experiment 1 . However, since it is known that DF performs well when full vision is provided (closed loop) and since we did not find any evidence that DF's performance depends crucially on the visibility of the target during the reaction time period, we decided to only include the OLM condition (dorsal processing) and $3 \mathrm{sD}$ condition (ventral processing). According to the perception-action model these are the two crucial vision conditions for testing the effects of delay on visuomotor performance.

Conditions were blocked and all participants performed the OLM and 3sD-conditions in alteration. Half of the control participants started with the EC-conditions and the other half with the EC+ conditions. Patient DF started with the EC- condition. The slot-orientations were presented randomly throughout each block with each orientation being presented 8 times (resulting in 40 trials per block). No instructions were given as to the speed of movement initiation and execution. The end of the posting movement was defined as the first frame in which the velocity of the card dropped below $0.05 \mathrm{~m} / \mathrm{sec}$ and the card was less than $2 \mathrm{~cm}$ away from the monitor screen. The data was analysed equivalently to Experiment 1.

\subsection{Results}

\subsubsection{Accuracy}


Our primary interest was in the accuracy with which the card orientation was matched to the orientation of the target slot in both the EC+ and EC- posting condition. Figure 4 shows the constant error, absolute error and variable error averaged across all slot orientations for DF and the control participants in all conditions. To test whether DF's performance was affected by the viewing condition, we applied a revised standardised difference test (Crawford et al., 2010). In the absence of environmental cues (EC-), the one-tailed t-tests revealed that DF showed similar absolute and constant errors as the control participants in the OLM-ECcondition, (constant error: $\mathrm{t}(9)=1.10, \mathrm{p}=.15$; absolute error: $\mathrm{t}(9)=0.67, \mathrm{p}=.26$ ), as well as in the 3sD-EC- condition, (constant error: $t(9)=0.45, p=.33$; absolute error: $t(9)=0.11, p=.46$ ). Moreover, the difference between both viewing conditions was similar for DF and the controls (constant error: $t(9)=1.07, p=.31$; absolute error $t(9)=0.7, p=.49$ ). Exactly the same pattern was observed for the variable error. Again, DF and the controls performed with a similar accuracy in the OLM-EC- condition, $\mathrm{t}(9)=0.03, \mathrm{p}=.49$, and the $3 \mathrm{sD}-\mathrm{EC}$ - condition, $\mathrm{t}(9)=0.19, \mathrm{p}=.43$, with a similar difference between both viewing conditions, $\mathrm{t}(9)=0.18$, $\mathrm{p}=.86$.

Interestingly, however, this pattern changed when environmental cues were present $(\mathrm{EC}+)$ and only the posting stimulus was removed from view. In these conditions, DF still showed a similar bias (constant error) as the controls in the OLM-EC+ condition, $\mathrm{t}(9)=0.70, \mathrm{p}=.25$, and the $3 \mathrm{sD}-\mathrm{EC}+$ condition, $\mathrm{t}(9)=1.11, \mathrm{p}=.15$ with a similar difference between those conditions, $\mathrm{t}(9)=1.14, \mathrm{p}=.28$ (see Figure 4B1). However, regarding the absolute error, we observed that DF was significantly worse than the controls in both the OLM-EC+ condition, $t(9)=2.03, p=.036$, and the $3 \mathrm{sD}-\mathrm{EC}+$ condition, $\mathrm{t}(9)=4.92, \mathrm{p}<.001$ (see Figure 4B2). Moreover, when environmental cues were present, DF's performance was significantly stronger affected by the $3 \mathrm{~s}$ delay than the performance of the controls, $t(9)=3.3, p=.004$. Therefore, DF's pattern of performance fulfils the criteria for a strong dissociation in the EC+ 
conditions according to Crawford's testing procedure (Crawford and Garthwaite, 2005). This finding is further supported by the analysis of the variable error. Again, DF's variable error is larger than the error of the controls in both viewing conditions (OLM-EC+: $t(9)=2.59, \mathrm{p}=.015$ and $3 \mathrm{sD}-\mathrm{EC}+\mathrm{t}(9)=4.24, \mathrm{p}=.001)$ with $\mathrm{DF}$ showing a marginally larger difference between both viewing condition than the controls, $\mathrm{t}(9)=2.08, \mathrm{p}=.034$ (one-tailed).

Furthermore, it is interesting to note that while control participants showed higher accuracy when environmental cues were present $(\mathrm{EC}+)$ for delay and non-delay conditions, DF tended to show no improvement in accuracy when environmental cues were present. Standardised difference-tests (Crawford and Garthwaite, 2005; Crawford et al., 2010) confirmed that the discrepancy in DF's performance between the EC- and the EC+ tasks was statistically different from that of the controls: in both viewing conditions for the variable errors (OLM$\mathrm{EC}+$ vs. OLM-EC-: $\mathrm{t}(9)=2.78, \mathrm{p}=.02$ and $3 \mathrm{sD}-\mathrm{EC}-$ vs. $3 \mathrm{sD}-\mathrm{EC}+: \mathrm{t}(9)=5.84, \mathrm{p}=.0003)$, and after $3 \mathrm{~s}$ delay for the absolute error $(3 \mathrm{sD}-\mathrm{EC}-\mathrm{vs}$. $3 \mathrm{sD}-\mathrm{EC}+\mathrm{t}(9)=4.73, \mathrm{p}=.001)$. The discrepancy between EC+ and EC- tasks did not reach significance for the absolute errors in the OLM-conditions (OLM-EC+ vs. OLM-EC-: $t(9)=2.06, p=.07)$. Moreover, the analysis revealed that DF's differences between the constant errors in the different environmental conditions were similar to those of the controls in both vision conditions (OLM-EC+ vs. OLM-EC-: $\mathrm{t}(9)=0.51, \mathrm{p}=.62$ and $3 \mathrm{sD}-\mathrm{EC}-\mathrm{vs} .3 \mathrm{sD}-\mathrm{EC}+: \mathrm{t}(9)=0.99, \mathrm{p}=.35)$. This finding suggests that even though DF performed generally less accurate and more variable than the controls in delay conditions in which environmental cues were available, she showed no strategic orientation bias different from that of the controls in any of those conditions.

\section{Insert Figure 4 about here}

Finally, we also examined the influence of the different visual conditions on the performance of the healthy control participants using a 2 (duration of vision: OLM vs. 3s-D) x 2 (presence 
of environmental cues: EC- vs. EC+) repeated-measures ANOVA. This revealed that, as expected, the factor duration of vision had a significant effect on movement accuracy, indicating that the accuracy of the movements decreased when visual feedback was reduced (absolute error: $\mathrm{F}(1,9)=6.23, \mathrm{p}=.03$; variable error: $\mathrm{F}(1,9)=45.82, \mathrm{p}<.001)$. However, there was no effect of duration of vision on the constant orienation error, $F(1,9)=0.8, p=.79$. Interestingly, we also found a significant influence of the factor presence of environmental cues indicating that healthy participants were more accurate in the $\mathrm{EC}+$ conditions in which only the target was removed from view than in the EC- conditions in which vision was removed completely (absolute error: $\mathrm{F}(1,9)=8.23, \mathrm{p}=.02$; variable error: $\mathrm{F}(1,9)=10.16$, $\mathrm{p}=.01)$. Regarding the constant error, participants tended to show a slight leftward bias when environmental cues were present $\left(-2.0^{\circ} \pm 0.4^{\circ}\right)$ but a slight rightward bias when vision was occluded completely $\left(3.9^{\circ} \pm 1.4^{\circ}\right), \mathrm{F}(1,9)=21.21, \mathrm{p}=.001$.

Furthermore, for the absolute error, we also observed a significant interaction effect, $\mathrm{F}(1,9)=$ $5.51, \mathrm{p}=.04$, indicating that the effect of delay was stronger in the EC+ conditions than in the EC- conditions. Note, that this interaction effect seems to be primarily driven by one of the control participants who showed a very high absolute orientation error in the OLM-ECcondition. If the data from this participant was removed from analysis the interaction effect was no longer significant $(p=.10)$. For the variable error, the interaction effect did not reach significance $(\mathrm{p}=.22)$.

\subsubsection{Reaction time (RT) and Movement time (MT)}

To investigate whether DF's movement planning or movement execution times differed from those of the controls (c.f. Table 2), RTs and MTs were averaged over all slot orientations for each visual feedback condition and for both the $\mathrm{EC}+$ and the EC- posting condition. We applied revised standardised difference tests (Crawford et al., 2010) to find out whether DF's 
RTs and MTs differed from those of the controls in the different posting conditions. In the absence of environmental cues (EC-), DF showed similar RTs and MTs as the control participants when vision was occluded at movement onset (OLM-EC- condition), (RT: $\mathrm{t}(9)=0.83, \mathrm{p}=.21 ; \mathrm{MT}: \mathrm{t}(9)=0.58, \mathrm{p}=.29)$, as well as after 3s Delay (3sD-EC- condition), (RT: $\mathrm{t}(9)=1.25, \mathrm{p}=.12 ; \mathrm{MT}: \mathrm{t}(9)=0.60, \mathrm{p}=.28)$. Moreover, the difference between both viewing conditions was similar for DF and the controls (RT: $\mathrm{t}(9)=1.00, \mathrm{p}=.35$; MT $\mathrm{t}(9)=0.06, \mathrm{p}=.95$ ). Similarly, in the EC+ conditions, RTs and MTs did not differ between DF and the control participants, neither for the OLM-conditions $(\mathrm{RT}: \mathrm{t}(9)=0.13, \mathrm{p}=.45$; $\operatorname{MT} \mathrm{t}(9)=0.55, \mathrm{p}=.30$ ) nor for the $3 \mathrm{~s}-\mathrm{D}$ condtions $(\mathrm{RT}: \mathrm{t}(9)=0.61, \mathrm{p}=.28 ; \mathrm{MT}: \mathrm{t}(9)=1.05, \mathrm{p}=.16)$. Again there was also a similar difference between the conditions for DF and the controls (RT: $t(9)=0.96, p=.36$; MT: $\mathrm{t}(9)=1.06, \mathrm{p}=.32)$. The data confirms that RTs as well as MTs were very similar for DF and the control participants across all tested conditions.

\section{Insert Table 2 about here}

\subsection{Discussion}

In this experiment, we investigated DF's posting performance in two different viewing conditions (OLM vs. 3s-D), and simultaneously varied the amount of environmental visual information available after target removal (EC+ vs. EC- conditions). In accordance with our findings from Experiment 1, we found that DF's performance was as accurate as the performance of the controls in the OLM and the 3s-D conditions when vision was occluded completely and participants had to rely solely on egocentric information. This finding also suggests that DF's preserved visuomotor performance after delay in Experiment 1 cannot be attributed to the fact that she received tactile feedback about the accuracy of her movements after each trial as no haptic feedback was present in the current experiment. It is interesting to contrast this finding with the recent observation that DF's grasping behaviour significantly deteriorates when haptic feedback is withdrawn (Schenk, 2012a). The differential effect of 
haptic feedback in grasping and posting suggests that the role and usefulness of haptic feedback strongly depends on the given task. In hindsight, the discrepancy between grasping and posting may however not be that surprising. Whereas in grasping, tactile feedback provides highly accurate feedback about the correct final position of the grasping fingers, the informative value of tactile feedback is much smaller in posting tasks. Tactile feedback in the form of the absence or presence of collisions with the posting board will only tell us whether we have been within the correct range of orientations or not. But such collision cannot discriminate between an error of say, 30 degrees and 130 degrees.

During the revision process of this manuscript, we became aware of the fact that some readers assume that Schenk (2012a) claimed that all instances of preserved action in DF rely on the presence of tactile feedback. However, such a generalised claim was not intended by Schenk (2012a) and would not have been supported by his findings. In his paper, Schenk (2012a) argued that tactile feedback can probably account for the perception-action dissociation between grasping and size-discrimination, but this is only one (albeit very important) example for a perception-action dissociation. A recent study by Whitwell et al. (2013) suggested that uninformative tactile feedback may be sufficient to restore DF's grasping ability to normal levels suggesting that, even in grasping, tactile feedback may play a limited role in guiding DF's visuomotor behaviour. In any case, the claim that tactile feedback is the one and only factor responsible for all perception-action dissociations in DF would seem quite implausible given that there are numerous examples of DF's visuomotor normalcy in the absence of informative tactile feedback (e.g. saccadic pointing: Rossit et al. (2010),; manual pointing: Carey et al. (2006); obstacle avoidance, Rice et al. (2006)). In our view, tactile feedback is only one of many cues that allow DF to compensate for her impaired visuo-spatial skills. In agreement with this line of argument, the current study provides further evidence that tactile feedback is not always critical for DF's visuomotor success. 
However, it will require further research to explore in detail which cue-combinations are relevant (and optimal) for performing a given motor task successfully.

Interestingly, we also observed that DF's performance was significantly less accurate than the performance of the control participants when only the target was removed from view while all other visual information remained available. In these $\mathrm{EC}+$ conditions, in which a combination of egocentric (moving limb) and allocentric (landmarks) information is available for movement control, DF's performance was clearly impaired both in the delay and in the OLM-condition. This is surprising, as according to the predictions of the perception-action model movements in the OLM-condition rely solely on visual information from the dorsal stream (Goodale et al., 2005) and should therefore be uncompromised in patient DF. Previous studies suggest that in situations in which visual landmarks are available, healthy participants rely on a combination of allocentric cues (visual landmarks) and egocentric cues (vision of the moving hand) in order to perform as accurately as possible (Conti and Beaubaton, 1980). Thus, DF's general impairment in the EC+ conditions might reflect not only her compromised ability to use environmental cues efficiently but also an inability to integrate both allocentric and egocentric cues for movement control. Note that in the EC- conditions the removal of environmental cues was associated with blocking participants' sight of their moving hand. That is, environmental cues and online visual feedback were always withdrawn simultaneously. Hence, the superior performance of healthy participants in conditions in which environmental cues were available may not just reflect a more efficient use of landmark information but possibly also of online visual feedback. In the General Discussion we will discuss this issue in more detail.

Looking at DF's deficit more closely it can be seen that her absolute posting error after $3 \mathrm{sec}$ delay was about $10^{\circ}$ in the $\mathrm{EC}+$ conditions, whereas the absolute posting error was smaller 
than $6^{\circ}$ for the control participants (see Figure 4). This finding seems to imply that even though DF still had a relatively accurate representation of the orientation of the target slot

after $3 \mathrm{sec}$, she was unable to match the high precision of the controls. By and large these findings are similar to the earlier studies testing DF's performance after delay in allocentric movement conditions (Milner et al., 1999; Rossit et al., 2010) and suggest that DF's behaviour has not significantly changed in this respect during the last 10 years. Our findings indicate that DF's delayed performance is only inferior to that of healthy controls when cues from the visual environment are available during the response period. The implications of these findings will be discussed in the next section.

\section{General Discussion}

In this study we re-examined the claim that an intact ventral visual stream is essential for delayed action (i.e. visually-guided actions where a delay is introduced between the offset of the visual target and the onset of the motor response). In Experiment 1, we obtained results that clearly challenge this claim. DF's performance in this experiment was of similar accuracy independent of whether the target was visible during movement programming or not (no difference between the OLM and OLS condition). This finding contradicts the real-time view of action (e.g., Goodale et al., 2005; Goodale et al., 2004; Westwood and Goodale, 2003) suggesting that the ventral stream is only bypassed in visuomotor tasks in which the target is visible at the moment of movement initiation. Accordingly, it is predicted that DF's ventral stream damage will leave movements in the OLM-condition unaffected but impairs movements in the OLS-condition. Contrary to this view DF's visuomotor performance was as accurate as the performance of the healthy control participants in both the OLM and the OLS conditions. Furthermore, the finding that DF's posting performance was undistinguishable from the performance of the controls even after a delay of $3 \mathrm{sec}$, is inconsistent with claims 
stating that she is generally unable to generate normal actions after longer delays (Goodale et al., 1994; Milner et al., 1999; Milner and Goodale, 2006; Rossit et al., 2010). According to the perception-action model (Milner and Goodale, 1995) it was suggested that movements after a delay primarily depend on visual input from the ventral stream, a structure that is largely damaged in DF (James et al., 2003). The finding that DF performed accurate posting movements in all vision conditions including delay-conditions shows that delayed visuomotor performance does not necessarily require ventral stream input. Instead it seems that in some conditions visual information from the dorsal stream is sufficient to guide actions in both immediate and delayed conditions. This view is consistent with recent neurophysiological, neuroimaging and psychophysical studies which provide cumulative evidence that dorsal cortical areas are not only active in the immediate but also in the delayed guidance of actions (Cohen et al., 2009; Connolly et al., 2003; Fiehler et al., 2011; Himmelbach et al., 2009; Murata et al., 2000; Rogers et al., 2009).

Additionally, we observed in Experiment 1 that DF's performance was considerably impaired when she was asked to pantomime a posting movement, even when pantomiming was performed in full-vision of hand and target. This is the first time that a dissociation between delayed movements and pantomime movements has been demonstrated in patient DF. This dissociation suggests that these two types of tasks do not necessarily involve the same visual cues and processing mechanisms. More precisely, our findings suggest that while pantomimed posting relies on the ventral stream (and allocentric processing), the same is not necessarily true for delayed movements - at least not in all conditions.

In Experiment 2, we examined factors that might explain why in our experiment (but not in previous studies) DF showed normal behaviour under delayed conditions. Firstly, by using 
the virtual-slot paradigm, i.e. a paradigm where posting success is no longer associated with a specific type of tactile feedback, we could rule out that she relies on tactile feedback to achieve normal performance under delayed conditions. Secondly, we observed that the availability of environmental cues can partly explain why DF performs worse than healthy participants under delayed conditions in some but not all experiments. That is, when environmental cues were available during and after the delay period, DF's posting performance was impaired relative to the control participants. While healthy participants perform more accurately when environmental cues were available, the same was not true for DF. Given that DF has significant problems in processing scene-based spatial relationships (allocentric information, see Carey et al., 2006; Dijkerman et al., 1998; Schenk, 2006), it is perhaps not surprising that she cannot exploit the spatial relation between a remembered target relative to still present visual landmarks. There is evidence that the significance of such allocentric cues in visuomotor control changes with delay, with landmark information becoming more important when the motor system has to rely on a memory trace of the visual target (Bridgeman et al., 1997; Hay and Redon, 2006; Obhi and Goodale, 2005). This might explain why DF often performs less accurately in delayed conditions in which visual landmarks remain available during the delay period.

However, it has to be noted that our findings do not provide unequivocal support for an account merely based on environmental cues. As we stated above, in our experiment, the withdrawal of environmental cues was associated with the simultaneous withdrawal of online visual feedback from the moving hand. Thus, it is possible that the superior performance of healthy participants in the EC+ conditions reflects a superior capacity to make use of online visual feedback. At present, this alternative account cannot be ruled out. However, there are some reasons suggesting that the environmental cue account is more likely than the visual 
feedback account. While there is good evidence to suggest that DF cannot perceive scenebased relations (and thus by extension will find it difficult to benefit from visual landmarks, see Carey et al., 2006; Dijkerman et al., 1998; Schenk, 2006), there is currently no evidence to suggest that DF is less efficient in using online visual feedback than healthy controls. Moreover, the results of our Experiment 1 also suggest that DF is using visual feedback as effectively as healthy participants: her performance with and without online visual feedback falls nicely with the normal range (CL vs. OLM-conditions). DF's performance deteriorates somewhat when visual feedback is withdrawn (difference between CL and OLM-conditions), but this performance decrement is very similar to the performance decrement observed for the healthy participants. This suggests that DF uses visual feedback in pretty much the same way and with comparable efficiency as the healthy controls. In contrast, her pattern of behaviour clearly dissociates from that of healthy controls when we compare the EC+ and EC- conditions in Experiment 2. Therefore, we suggest that it is more likely that the dissociation between DF and healthy controls in these tasks is driven by a difference in the ability to use environmental cues efficiently rather than by a difference in the ability to use online visual feedback.

Finally, our findings are also well in line with the observation that DF only performs normal movements in very specific and well defined situations. For example, it has been shown that targets have to be presented binocularly (Dijkerman et al., 1996; Marotta et al., 1997) and in the central visual field (Hesse et al., 2012) in order to observe normal visuomotor behaviour in patient DF. Schenk (2010) suggested that the limitation of DF to generate normal movements only in natural situations might be best explained by the fact that natural situations are rich in sensory cues, meaning that in such conditions, motor behaviour can rely on many equally valid sources of visual information. Once this sensory redundancy is reduced, DF will frequently face problems due to the fact that her brain damage has left her 
with seriously restricted access to visual information. This means that while healthy participants can easily switch from one visual strategy and one source of visual information to another strategy or source, DF's range of options is much more restricted. This general framework could also explain why delay impairs DF's performance only in some tasks. According to this reasoning, delay will only lead to abnormal behaviour if it forces participants to use cues (presumably allocentric in nature) which can be fruitfully exploited by healthy individuals but not by DF. This interpretation is further supported by the pattern of findings obtained in our current study.

Before we conclude, we should address two alternative explanations that were suggested to us during the review process. One reviewer argued that our findings might be due to the fact that we blocked the different conditions in our experiment. Accordingly DF and the other participants always knew in advance whether to expect a delay or not. It was argued that this allowed DF to use a strategy. She may for example have used motor imagery to achieve normal motor performance in the delayed condition. This is a possibility for which there is evidence from an earlier study suggesting that DF's performance in a perceptual task improves when she is instructed to use motor imagery (Dijkerman and Milner, 1997). However, unless explicit instructions for the use of motor imagery are given it is hard to know whether DF will make use of this strategy or not. In our case we certainly did not instruct DF to use motor imagery. Nevertheless after having participated in numerous experiments she may have learned that motor imagery is very useful and may therefore employ it spontaneously. However, one could expect that she would not apply this strategy selectively to the delayed condition, but also to other difficult conditions. Interestingly, our results indicate that DF did not use motor imagery in the pantomime conditions in which her performance was still significantly impaired. This seems to suggest that DF either does not employ the motor imagery strategy spontaneously or that this strategy does not always help. 
For these reasons we think it is unlikely that motor imagery explains how DF achieves normal posting performance under delayed conditions.

However, motor imagery is not the only strategy that DF could have used. It is also possible that DF had a subvocal verbal code for each of the different posting responses and selected the appropriate response code as soon as the visual target stimulus appeared (e.g., slot at 2 o'clock). In principle, such a strategy should have a big advantage. During the delay she only needs to remember the verbal code, which presumably she can hold in her intact verbal memory and does not need to rely on a visuospatial memory of the target configuration (which depends on the damaged ventral stream). In short, using a verbal code might therefore help her to circumvent her visuo-spatial memory deficit. Yet, there is a problem with this strategy. Where does the visual information that is used to select the appropriate response code come from? According to the perception-action model, this information must come from DF's dorsal stream. But proponents of the perception-action model have always insisted that dorsal visual information can only be used to guide direct motor responses, i.e. responses for which the characteristics of the target stimulus directly map to the aspects of the motor response (e.g. object size maps to grip aperture size in grasping). Making this assumption is quite essential for proponents of the perception-action model, as it would otherwise be quite mysterious why visual information in the dorsal stream cannot be used by DF to guide arbitrary motor responses in so-called perceptual tasks (such as button presses). The strategy of using verbal codes would, however, require that the dorsal stream is capable of such arbitrary visual-response mappings. Hence, we feel that the idea of DF using a verbal strategy to circumvent the consequences of the introduced delay is difficult to reconcile with the classic view of the perception-action model. Ultimately it requires, however, an empirical test to decide whether DF's ability to produce normal posting performance under delayed conditions depends on the blocked presentation of vision conditions. 
Carey and his colleagues faced a similar problem when they tested the blindsight patient DB (Carey et al., 2012). They found that DB's ability to point or make a saccade to visual targets in his blind field was not abolished when a delay was inserted between target presentation and response onset. In principle, DB may have coded the appropriate response at the time of target presentation. To address this possibility Carey et al. (2012) used an elegant design. They randomly presented the visual target either late during the trial interval (without a delay between target presentation and response cue) or early in the trial interval (with a $4 \mathrm{sec}$ delay between target presentation and response cue). This manipulation ensured that DB could not predict whether he was allowed to respond immediately or not. A similar design could be used to test whether DF is only able to produce normal posting performance under delayed conditions when she is able to predict the occurrence of a delay. We collected some pilot-data in our lab in which we asked DF to perform posting movements to a monitor in randomly presented CL, OLM and OLS-viewing conditions and found that she is still able to perform as accurately as the controls in all viewing conditions (all $\mathrm{p}>.17$ ) even though the OLScondition would require ventral stream involvement according to the real-time view of action (Goodale et al., 2005).

\section{Conclusions}

We found that delay affects DF's performance in some tasks but not in others. Importantly, any effects of delay on DF's performance were only found in conditions in which environmental cues and online visual feedback remained available. Furthermore, DF was able to perform accurate posting movements independently of whether tactile feedback was provided at the end of the movement or not. Based on these results, we suggest that the availability of environmental cues during the delay period explains why healthy individuals perform better than DF in certain delayed movement tasks. Such an interpretation is not that 
different from Milner and Goodale's (1995/2006) original claim that delay forces the visuomotor system to rely on allocentric and thus ventral information. However, there are some relevant differences which are worth re-iterating. We argue that the time scale of processes and representations in dorsal and ventral streams are not necessarily dissimilar. Furthermore, we doubt the assumption that introducing a delay leads to a mandatory switch from dorsal to ventral stream processing. Instead, as shown in the current study, delay does not always lead to abnormal behaviour when ventral areas are damaged (in the case of DF). Performance rather depends on the given visuomotor task, the availability of environmental (allocentric) cues and/or the presence of online visual feedback. Clearly, our findings so far refer to just one visuomotor paradigm and it remains to be seen whether similar results can be obtained in other tasks. However, the results obtained for the letter-posting task alone already show that the general assumption of critical ventral stream involvement in all delayed visually-guided actions can no longer be upheld and that an alternative explanation is required. We argue that an explanation that stresses the natural sensory redundancy of visuomotor tasks, and that emphasises DF's increased reliance on this redundancy (Schenk, 2010), can easily account for the effects of delay on DF's behaviour without having to make a special provision for delayed behaviour. 


\section{Acknowledgement}

The authors would like to thank DF for participating in all our experiments with great patience. We also would like to thank Dr. David Carey for his very helpful and insightful comments on an earlier draft of this manuscript. This work was partly funded by a postdoctoral research fellowship awarded to Constanze Hesse by the German research council (DFG/HE 6011/1-1). 


\section{References}

Brainard, D.H. The psychophysics toolbox. Spatial Vision 10 (4): 433-436, 1997.

Bridgeman, B., Peery, S., and Anand, S. Interaction of cognitive and sensorimotor maps of visual space.

Perception and Psychophysics 59 (3): 456-469, 1997.

Carey, D.P., Dijkerman, H.C., Murphy, K.J., Goodale, M.A., and Milner, A.D. Pointing to places and spaces in a patient with visual form agnosia. Neuropsychologia 44 (9): 1584-1594, 2006.

Carey, D.P., Trevethan, C.T., Weiskrantz, L., and Sahraie, A. Does delay impair localisation in blindsight? Neuropsychologia 50 (14): 3673-3680, 2012.

Cohen, N.R., Cross, E.S., Tunik, E., Grafton, S.T., and Culham, J.C. Ventral and dorsal stream contributions to the online control of immediate and delayed grasping: A TMS approach. Neuropsychologia 47 (6): 1553-1562, 2009.

Connolly, J.D., Andersen, R.A., and Goodale, M.A. FMRI evidence for a 'parietal reach region' in the human brain. Experimental Brain Research 153: 140-145, 2003.

Conti, P. and Beaubaton, D. Role of structured visual field and visual reafference in accuracy of pointing movements. . Perceptual and Motor Skills 50: 239-244, 1980.

Crawford, J.R. and Garthwaite, P.H. Testing for suspected impairments and dissociations in single-case studies in neuropsychology: Evaluation of alternatives using Monte Carlo simulations and revised tests for dissociations. Neuropsychology 19 (3): 318-331, 2005.

Crawford, J.R., Garthwaite, P.H., and Porter, S. Point and interval estimates of effect sizes for the case-controls design in neuropsychology: Rationale, methods, implementations, and proposed reporting standards. Cognitive Neuropsychology 27 (3): 245-260, 2010.

Crawford, J.R. and Howell, D.C. Comparing an individual's test score against norms derived from small samples. Clinical Neuropsychologist 12 (4): 482-486, 1998.

Dijkerman, H.C. and Milner, A.D. Copying without perceiving: Motor imagery in visual form agnosia. Neuroreport 8 (3): 729-732, 1997.

Dijkerman, H.C., Milner, A.D., and Carey, D.P. The perception and prehension of objects oriented in the depth plane.1. Effects of visual form agnosia. Experimental Brain Research 112 (3): 442-451, 1996.

Dijkerman, H.C., Milner, A.D., and Carey, D.P. Grasping spatial relationships: Failure to demonstrate allocentric visual coding in a patient with visual form agnosia. Consciousness and Cognition 7: 424-437, 1998. Elliott, D., Calvert, R., Jaeger, M., and Jones, R. A visual representation and the control of manual aiming movements. Journal of Motor Behavior 22 (3): 327-346, 1990.

Fiehler, K., Bannert, M.M., Bischoff, M., Blecker, C., Stark, R., Vaitl, D., Franz, V.H., and Rosler, F. Working memory maintenance of grasp-target information in the human posterior parietal cortex. Neuroimage 54 (3): 2401-2411, 2011.

Franz, V.H. The Optotrak Toolbox. http://www.allpsych.unigiessen.de/vf/OptotrakToolbox/, 2004.

Goodale, M.A., Jakobson, L.S., and Keillor, J.M. Differences in the visual control of pantomimed and natural grasping movements. Neuropsychologia 32: 1159-1178, 1994.

Goodale, M.A., Kroliczak, G., and Westwood, D.A. Dual routes to action: Contributions of the dorsal and ventral streams to adaptive behavior. Progress in Brain Research 149: 269-283, 2005.

Goodale, M.A. and Milner, A.D. Separate visual pathways for perception and action. Trends in Neurosciences 15: 97-112, 1992.

Goodale, M.A., Milner, A.D., Jakobson, L.S., and Carey, D.P. A neurological dissociation between perceiving objects and grasping them. Nature 349: 154-156, 1991.

Goodale, M.A. and Westwood, D.A. An evolving view of duplex vision: Separate but interacting cortical pathways for perception and action. Current Opinion in Neurobiology 14: 203-211, 2004.

Goodale, M.A., Westwood, D.A., and Milner, A.D. Two distinct modes of control for object--directed action. Progress in Brain Research 144: 131-144, 2004.

Hay, L. and Redon, C. Response delay and spatial representation in pointing movements. Neuroscience Letters 408 (3): 194-198, 2006.

Hesse, C., Ball, K., and Schenk, T. Visuomotor performance based on peripheral vision is impaired in the visual form agnosic patient DF. Neuropsychologia 50 (1): 90-97, 2012.

Hesse, C. and Franz, V.H. Memory mechanisms in grasping. Neuropsychologia 47 (6): 1532-1545, 2009.

Hesse, C. and Franz, V.H. Grasping remembered objects: Exponential decay of the visual memory. Vision Research 50 (24): 2642-2650, 2010.

Himmelbach, M. and Karnath, H.O. Dorsal and ventral stream interaction: Contributions from optic ataxia. Journal of Cognitive Neuroscience 17: 632-640, 2005.

Himmelbach, M., Nau, M., Zündorf, I., Erb, M., Perenin, M.T., and Karnath, H.O. Brain activation during immediate and delayed reaching in optic ataxia. Neuropsychologia 47 (6): 1508-1517, 2009. 
$\mathrm{Hu}, \mathrm{Y}$. , Eagleson, R., and Goodale, M.A. The effects of delay on the kinematics of grasping. Experimental Brain Research 126: 109-116, 1999.

James, T.W., Culham, J., Humphrey, G.K., Milner, A.D., and Goodale, M.A. Ventral occipital lesions impair object recognition but not object-directed grasping: an fMRI study. Brain 126: 2463-2475, 2003.

Kleiner, M. Visual stimulus timing precision in Psychtoolbox-3: Tests, pitfalls and solutions. Perception 39: 189-189, 2010.

Krigolson, O. and Heath, M. Background visual cues and memory-guided reaching. Human Movement Science 23 (6): 861-877, 2004.

Lacquaniti, F., Perani, D., Guigon, E., Bettinardi, V., Carrozzo, M., Grassi, F., Rossetti, Y., and Fazio, F. Visuomotor transformations for reaching to memorized targets: A PET study. Neuroimage: 129-146, 1997. Lemay, M., Bertram, C.P., and Stelmach, G.E. Pointing to and egocentric an allocentric remembered target. Motor Control 8 (1): 16-32, 2004.

Marotta, J.J., Behrmann, M., and Goodale, M.A. The removal of binocular cues disrupts the calibration of grasping in patients with visual form agnosia. Experimental Brain Research 116: 113-121, 1997.

Milgram, P. A spectacle-mounted liquid-crystal tachistoscope. Behavior Research Methods, Instruments, \& Computers 19 (5): 449-456, 1987.

Milner, A.D., Dijkerman, H.C., and Carey, D.P. Visuospatial processing in a pure case of visual-form agnosia. In Burgess, N., Jeffery, K.J., and O'Keefe, J. (Eds.), The hippocampal and parietal foundations of spatial cognition. Oxford University Press: Oxford, 1999. 443-466.

Milner, A.D., Dijkerman, H.C., Pisella, L., McIntosh, R.D., Tilikete, C., Vighetto, A., and Rossetti, Y. Grasping the past. delay can improve visuomotor performance. Current Biology 11 (23): 1896-1901, 2001.

Milner, A.D., Ganel, T., and Goodale, M.A. Does grasping in patient DF depend on vision? Trends in Cognitive Sciences 16 (5): 256-257, 2012.

Milner, A.D. and Goodale, M.A. The visual brain in action. Oxford: Oxford University Press, 1995.

Milner, A.D. and Goodale, M.A. The visual brain in action., 2nd ed. Oxford University Press: Oxford, 2006.

Milner, A.D., Perrett, D.I., Johnston, R.S., Benson, P.J., Jordan, T.R., Heeley, D.W., Bettucci, D., Mortara, F., Mutani, R., and Terazzi, E. Perception and action in visual form agnosia. Brain 114: 405-428, 1991.

Murata, A., Gallese, V., Luppino, G., Kaseda, M., and Sakata, H. Selectivity for the shape, size, and orientation of objects for grasping in neurons of monkey parietal area AIP. Journal Of Neurophysiology 83 (5): 2580-2601, 2000.

Obhi, S.S. and Goodale, M.A. The effects of landmarks on the performance of delayed and real-time pointing movements. Experimental Brain Research 167 (3): 335-344, 2005.

Rice, N.J., McIntosh, R.D., Schindler, I., Mon-Williams, M., Démonet, J.F., and Milner, D.A. Intact automatic avoidance of obstacles in patients with visual form agnosia. Experimental Brain Research 174: 176-188, 2006. Rogers, G., Smith, D., and Schenk, T. Immediate and delayed actions share a common visuomotor transformation mechanism: A prism adaptation study. Neuropsychologia 47 (6): 1546-1552, 2009.

Rossetti, Y. Implicit short-lived motor representations of space in brain damaged and healthy subjects.

Consciousness and Cognition 7 (3): 520-558, 1998.

Rossit, S., Szymanek, L., Butler, S.H., and Harvey, M. Memory-guided saccade processing in visual form agnosia (patient DF). Experimental Brain Research 200 (1): 109-116, 2010.

Schenk, T. An allocentric rather than perceptual deficit in patient DF. Nature Neuroscience 9: 1369-1370, 2006. Schenk, T. Visuomotor robustness is based on integration not segregation. Vision Research 50 (24): 2627-2632, 2010.

Schenk, T. No Dissociation between Perception and Action in Patient DF When Haptic Feedback is Withdrawn. Journal of Neuroscience 32 (6): 2013-2017, 2012a.

Schenk, T. Response to Milner et al.: Grasping uses vision and haptic feedback. Trends in Cognitive Sciences 16 (5): 258-259, 2012b.

Westwood, D.A. and Goodale, M.A. Perceptual illusion and the real-time control of action. Spatial Vision 16 (34): 243-254, 2003.

Westwood, D.A., Heath, M., and Roy, E.A. The accuracy of reaching movements in brief delay conditions. Canadian Journal of Experimental Psychology 55 (4): 304-310, 2001.

Westwood, D.A., Heath, M., and Roy, E.A. No evidence for accurate visuomotor memory: systematic and variable error in memory-guided reaching. Journal of Motor Behavior 35 (2): 127-133, 2003.

Whitwell, R.L. and Buckingham, G. Reframing the action and perception dissociation in DF: haptics matters, but how? Journal of Neurophysiology 109 (3): 621-624, 2013.

Whitwell, R.L., David Milner, A., Cavina-Pratesi, C., Byrne, C.M., and Goodale, M.A. DF's visual brain in action: The role of tactile cues. Neuropsychologia in press, 2013.

Whitwell, R.L. and Goodale, M.A. Updating the programming of a precision grip is a function of recent history of available feedback. Experimental Brain Research 194 (4): 619-629, 2009.

Whitwell, R.L., Lambert, L.M., and Goodale, M.A. Grasping future events: explicit knowledge of the 
availability of visual feedback fails to reliably influence prehension. Experimental Brain Research 188 (4): 603$611,2008$. 


\section{Figure Legends}

Figure 1: Experiment 1: Event sequences for closed-loop (CL), open-loop after movement initiation (OLM), OL after start-signal (OLS), 3 sec delay (3s-D) conditions. The gray shaded bars indicate the time-interval during which target and hand are invisible. Further details are given in the text.

Figure 2: Experimental setup used in the posting task in Experiment 1. A) posting movement B) pantomime movement

Figure 3: Experiment 1: Orientation error of the card (averaged over all slot orientations) as a function of target visibilty for patient DF (filled circle) and ten control participants (open diamonds). A: constant orientation error, B: absolute orientation error, C: variable orientation error. The dashed line indicates the maximum orientation error which would still allow a successful posting through the slot (diagonally).

Figure 4: Experiment 2: Posting accuracy as a function of target visibility for patient DF (filled symbols) and ten control participants (open symbols). A1-A3: ECcondition (no visibility of hand and surrounding), B1-B3: EC+ condition (hand and surrounding remain visible). Posting movements were performed toward a slot presented on the screen of a monitor providing no haptic feedback about posting accuracy at the end of the movement. The dashed line indicates the maximum orientation error which would still allow a successful posting through the slot (diagonally). 


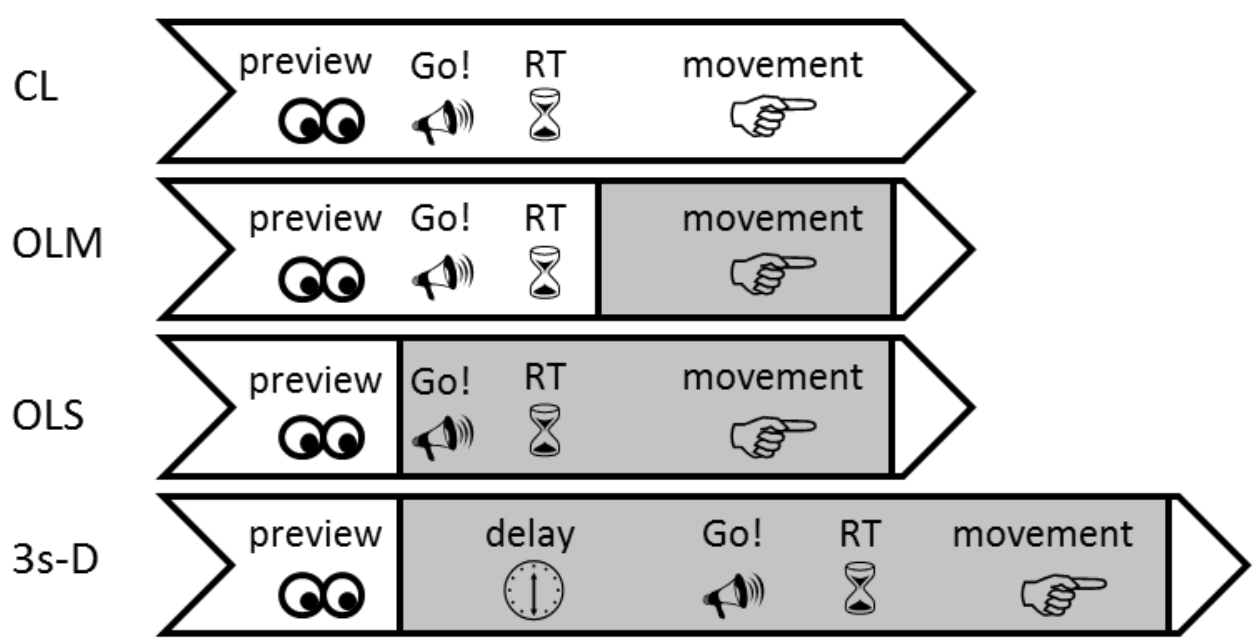

Figure 1 
A:

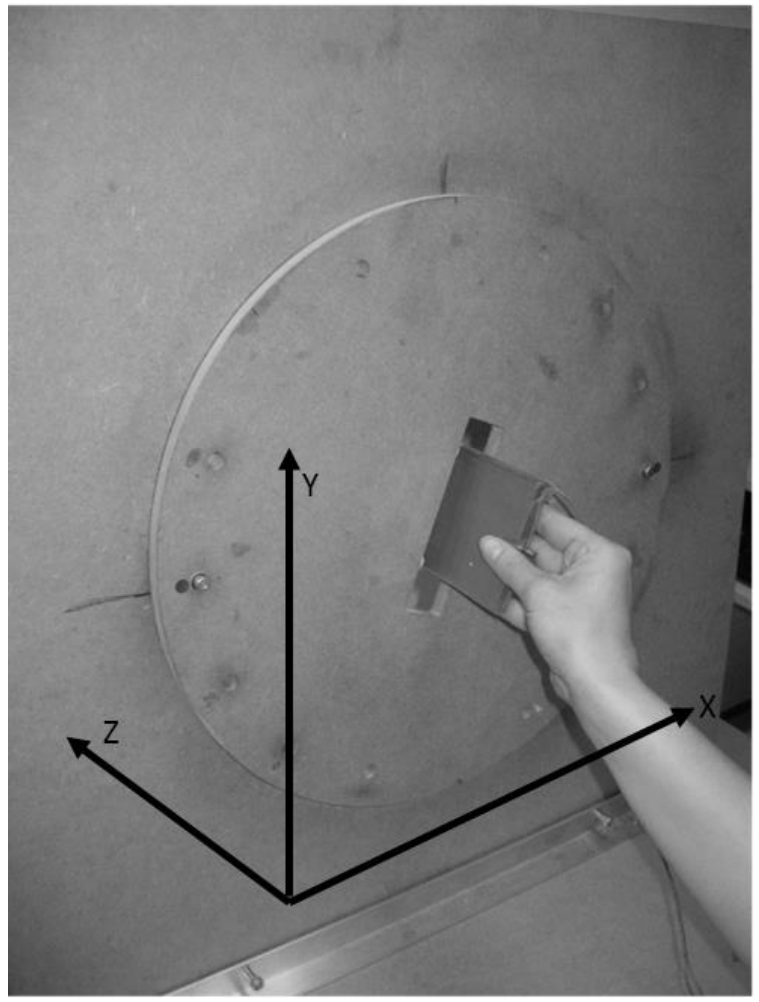

Figure 2 
A:

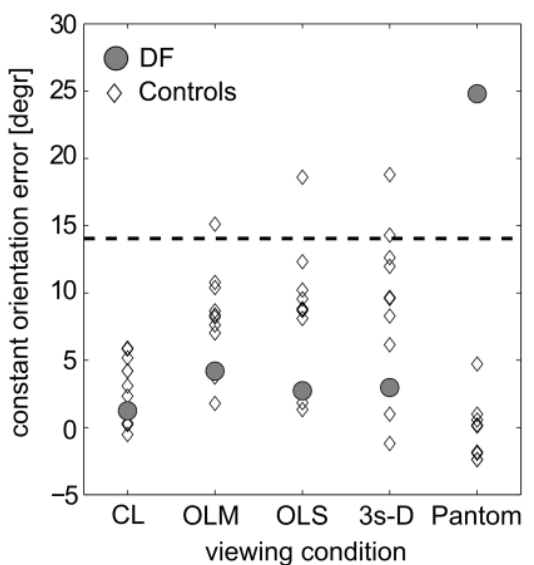

B:

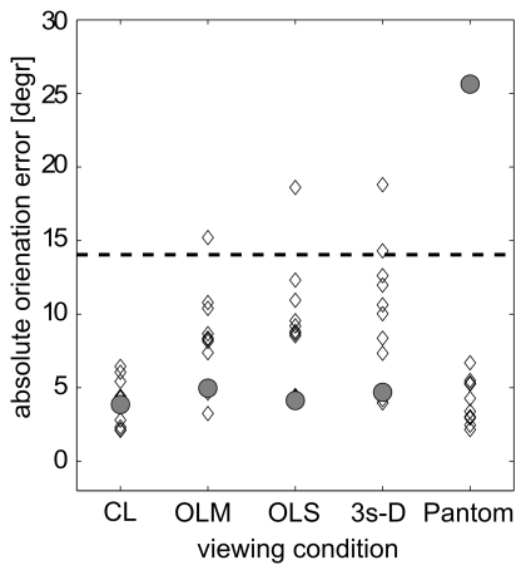

C:

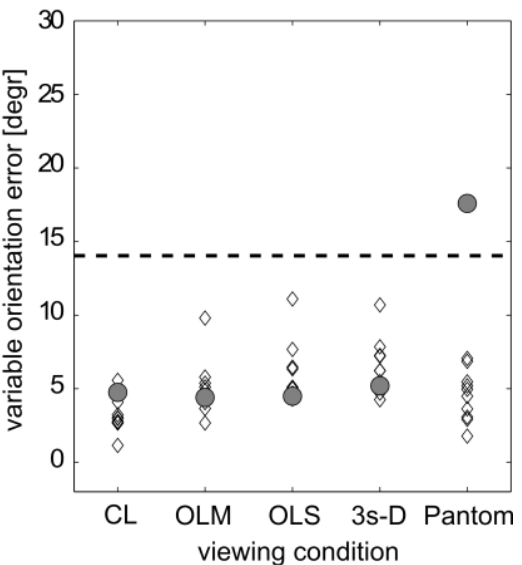

Figure 3 
A1

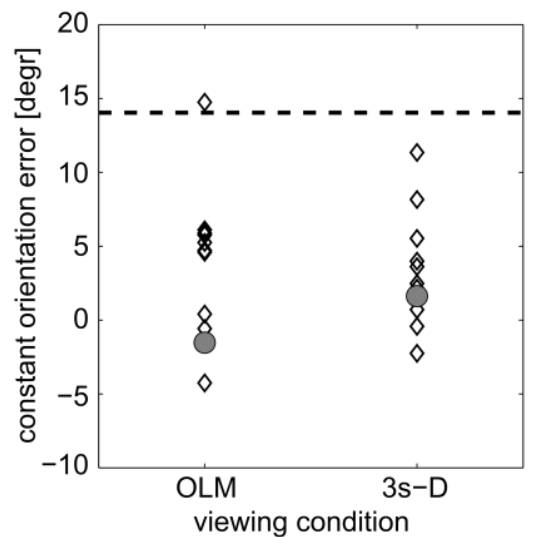

B1

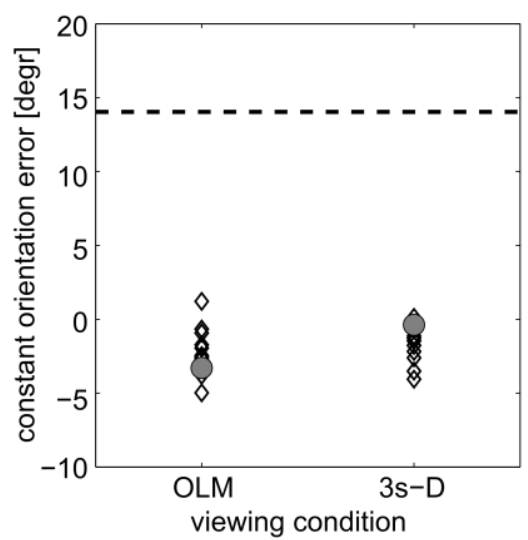

A2

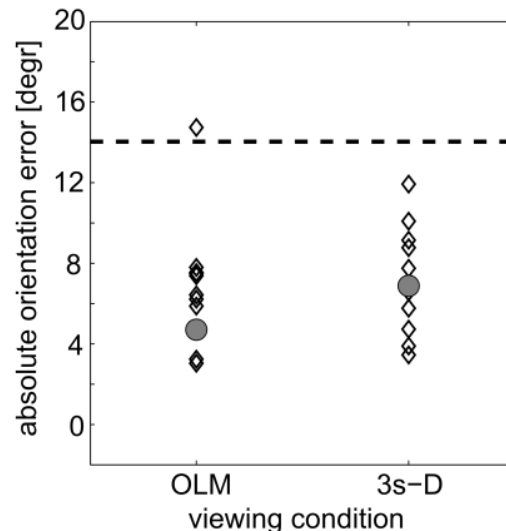

B2

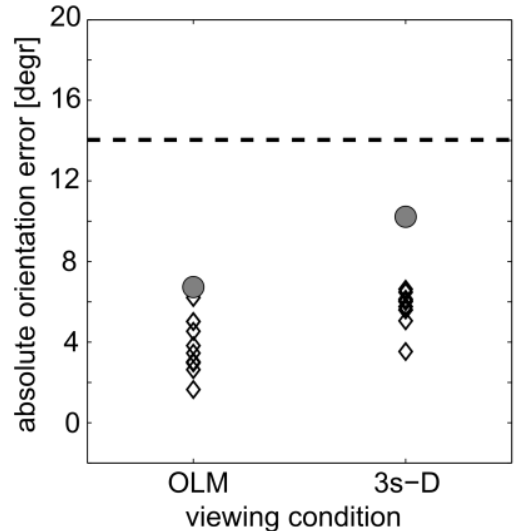

A3

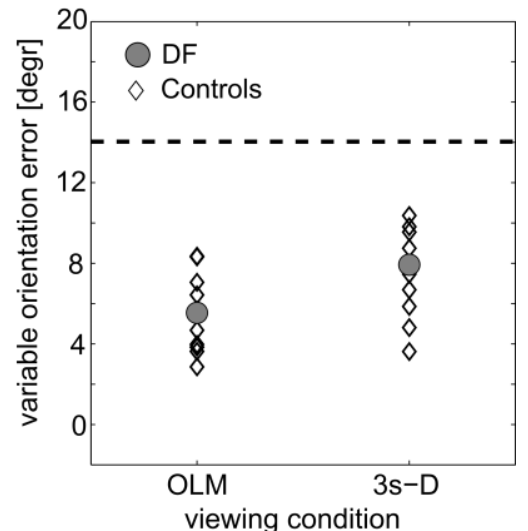

B3

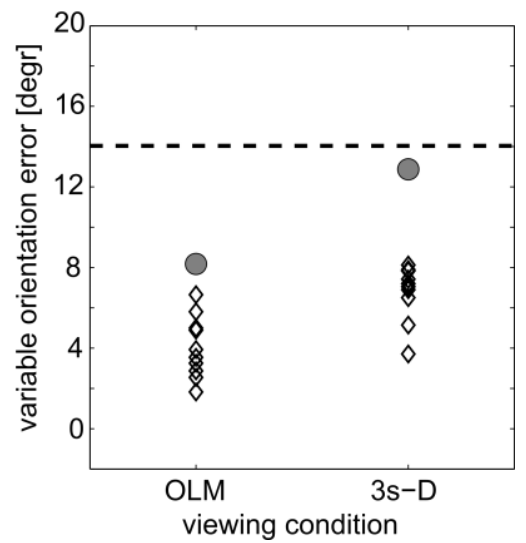

Figure 4 
Table 1: Experiment 1: Mean values for the RTs and MTs in the different fixation conditions (in $\mathrm{msec}$ ). SDs are reported in parenthesis.

\begin{tabular}{|c|c|c|c|c|}
\hline & \multicolumn{2}{|c|}{$\mathrm{RT}$} & \multicolumn{2}{|c|}{ MT } \\
\hline & $\overline{\mathrm{DF}}$ & Controls & $\overline{\mathrm{DF}}$ & Controls \\
\hline \multicolumn{5}{|c|}{ vision condition } \\
\hline $\mathrm{CL}$ & $257(115)$ & 358 (107) & $1019(98)$ & 857 (172) \\
\hline OLM & $325(129)$ & $432(158)$ & $1324(420)$ & $1054(318)$ \\
\hline OLS & $363(150)$ & $260(77)$ & $1207(232)$ & $1070(342)$ \\
\hline $3 s-D$ & $353(100)$ & $316(94)$ & $1251(334)$ & $1164(300)$ \\
\hline
\end{tabular}


Table 2: Experiment 2: Mean values for the RTs and MTs in the different viewing conditions (in $\mathrm{msec}$ ). SDs are reported in parenthesis.

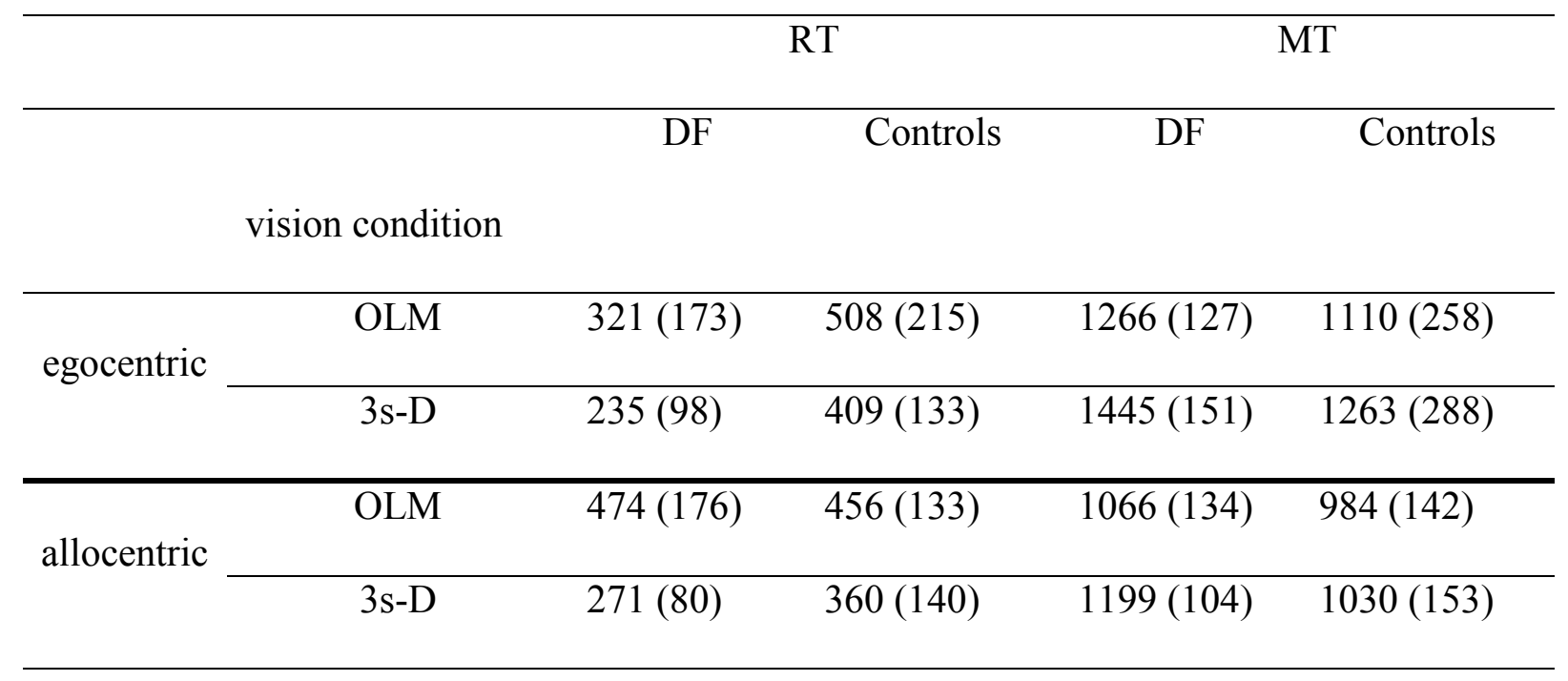

